\section{ambient SCIENCE \\ Vol. 05(Sp1):28-33 \\ Year 2018}

\title{
Impact of Personal Training and Educational Booklet Distribution Strategies on the Anxiety and Regulating Sexual Function in Infertile Women: A Case Study of Iran
}

\section{Safieh Jamali', NaderSharifi', Shiva Bigizadeh, Abdolali Sepidkar ${ }^{2 *}$}

'Research Center for Social Determinants of Health, Jahrom University of Medical Sciences, Jahrom , Iran

${ }^{2}$ Department of General Surgery, Jahrom University of Medical Sciences, Jahrom, Iran

Study Area: Jahrom, Iran

Coordinates: $28^{\circ} 30^{\prime} 0^{\prime \prime} \mathrm{N}$; $53^{\circ} 33^{\prime} 38^{\prime \prime} \mathrm{E}$

Key words: Face to face teaching, State-trait Anxity Invertory Female sexual function index

The study was approved by the Ethics Committee of the Jahrom University of Medical Sciences (JUMS.REC.1390.036).

\section{Abstract}

Infertility acts as a psychological blow in a couple's life. It also reduces sexual satisfaction in infertile women. The present study aimed to compare the effect of infertility treatment while face-to-face and educational booklet teaching methods on anxiety levels and sexual function among infertile women by conducting a clinical trial on 111 randomly selected infertile women. Total subjects were grouped into three categories of 37 each. Those who received face-to-face training and/or booklet training were placed in experimental groups while the control group received no training. The data collection instruments consisted of a demographic questionnaire, the Female Sexual Function Index, and Spiel Berger Anxiety Inventory. In personal training group, sexual function score and anxiety score altered significantly, but in training group by the booklet distribution, the sexual function score increased whereas anxiety score decreased. After the intervention, there were statistically significant differences also revealed between groups of intervention and control in five domains of sexual function; desire, arousal, lubrication, orgasm, and dyspareunia. Finally, methods of infertility treatment by experienced specialists either by personal training or via distributing educational booklet both were seen to be effective for infertile women.

Ramezanzadeh et al. (2004) the prevalence of anxiety was reported $86.8 \%$, similarly prevalence mentioned sexual dysfunction in infertile women as to be $87.1 \%$ (Jamali et al., 2013)..Few studies in Iran reported this prevalence to be 74.5\% to 56\% (Mirblouk et al., 2016; Pakpour et al., 2012). Women under stress fail to express their feelings and desire properly, as cortisol prevents oxytocin in female's brain and inhibits the willingness for the sexual and physical relationship (Kring \& Johnson, 2017; Kashdan et al., 2011). Kashdan et al. (2011) established a relationship between social anxiety and sexual relationships and was found to be negligible among women and reverse among men. They affirmed that people with anxiety, compared to those without anxiety, experience dysfunction in sexual performance. Given the high prevalence of anxiety and sexual dysfunction in infertile women, it is needed that, in addition to infertility treatments, approaches to improve their psychological status and sexual infertility must be reported in between 25 and $60 \%$ (Hamid, 2011). In a study by

*Corresponding Author: aali-sepid@yahoo.com 
considered. Studies in the field of the quality of life in infertile women had considered counseling, education and psychological intervention necessary to help them (Choobforoushzadeh et al., 2010). Valuable and useful impacts of education on patients have been described in several studies which seen to increase patients' satisfaction level, improved quality of life, relieving patients' anxiety, reduced complications, and increased patients' participation in health care programs and independence in carrying out the activities of daily living (Bastable, 2013). There are different methods for training which include: computer task, distributing training kit, group discussion, lectures, asking questions, documentary movies, exercises, simulations, and drama play (Mousavi et al., 2011).

Various studies have reported the effectiveness of sex education on marital satisfaction. In this regard, results obtained by Shahkarami et al., (2014) showed that sex education increases significantly the sexual intimacy in married women, similarly Ebrahimipour et al., (2013) also stated that the sex education significantly increases women's knowledge, attitude and sexual function in the intervention group. The study of Yusef-Zadeh et al. (2014) reported that the sex education based on religious teachings has a significant effect on marital adjustment of married women. Tai \& Paulson (2013) recognized the education as an effective element in increasing marital compatibility, how the couples communicated with each other improved significantly were much more satisfied with each other. It also significantly reduced their marital conflicts and their marital adjustment had increased. Onat \& Beji (2012) while exploring the quality of life and marital relationships in infertile couples stated that since the stress caused by the inability of couples to have children negatively affects their relations, emotion-centered education and effective sexual relations have a significant influence on improving couples' sexual relationships.Tavakolizadeh \& Hajivosogh (2012) also showed that educational, cognitive-behavioral therapy on women's marital satisfaction has increased marital satisfaction significantly in women with low sexual desire. Paying attention to the psychological needs of infertile couples is an essential part in the success of infertility treatment, because it can affect the relationship between the couple even during their treatment. Besides, one of the factors influencing satisfaction in sexual relations is their familiarity with sexual issues. One of the problems that Iranian society is facing today is the lack of adequate information on sexual issues and incorrect attitudes and beliefs towards it. Neverthless, most of the studies conducted on anxiety and sexual dysfunction in infertile women showed a high prevalence of this disorder and the lack of attention towards the educational needs of this group. Most of the studies pointed out the effect of intervention on anxiety and sexual function, but no study to compare the face-to-face (personal) training and the impact of relevant booklet distribtion on the anxiety and sexual function in infertile women. Hence, in this study, we aimed to investigate the efficacy of personal training and training through the booklet distribution on the anxiety and sexual function in infertile women

\section{Methodology:}

This study is a three-group randomized clinical trial study, possessing the pre-test and post-test on infertile women referring to an infertility clinic in Jahrom. The formula used was based on (Bahadorani et al., 2015). Following the protocol, the sample size was 33 , and, by adding $10 \%$ for drop-outs, the sample size increased to 37 (Bahadorani et al., 2015).

Through random assignment method, total 111 people were selected by easy sampling and then were divided into three groups of 37 subjects each, i) personal (face to face) teaching, ii) teaching through booklets distribution and iii) control. Samples were placed in experimental and control groups using a table of random numbers. Next, the samples were asked to complete a demographic questionnaire, FSFI questionnaire and anxiety based questionnaire. Further, the designed intervention plan was implemented for the two groups. Groups were synchronized in terms of variables (age, age difference with wife, husband's age, education and occupation).

The inclusion criteria were: not having any formal sex education, not suffering from a chronic disease, enjoying regular sexual intercourse with one's husband over the past four weeks prior to the study, not having a psychological disorder or Physical diseases (e.g. diabetes, rheumatoid arthritis, depression, etc.) that one knows of, not using drugs that affect one's sexual function (e.g. blood fatlowering drugs, cimetidine, digoxin, progesterone, and anti-depression drugs), speaking Farsi, and being Iranian.

In the personal training group, the instruction was provided by trainers based on individual learning during 8 sessions (2 times per week over 4 weeks) for 45 minutes. During training also, questions and answers between the instructor and the listener was a part whenever required. In addition to the researcher, two additional trained trainers were also used. To fix the time for the meetings, each of the targetted centers was given the schedule of meeting attendance also was reminded via telephone.

In compliance with research ethics, once the questionnaires were collected at the end of the study, educational pamphlets on sex education and infertility and the researcher's telephone number were made available to the women in the control group so that they could contact the researcher and ask for counsel like the participants in the intervention group. The instruments used in the study were FSFI and Spiel Berger inventory.

Female sexual function index: FSFI questionnaire was 
developed by Rosen et al. (200o) which consists of 19 questions investigating the subjects in 6 points (domain); sexual desire, sexual arousal, lubrication, orgasm, sexual satisfaction, and pain during intercourse. The given questions were scored based; $0 / 1-5$ scoring system and the score of each point was calculated through summing up the scores of that domain's questions and multiplying the obtained number by the multiplier factor of that domain. It should be mentioned that sexual desire was covered by questions 1 and 2, excitement by the sum of questions 3, 4, 5, and 6 , lubrication by adding questions $7,8,9$, and 10 , orgasm by the sum of questions 11, 12, and 13, sexual satisfaction by adding questions 14,15 , and 16 , and pain by summing up questions 17,18 , and 19 . In addition, multiplier factors of $0.6,0.4$, and 0.3 were used for points including 2 , 3 , \& 4 questions, respectively. In general, each point was having a minimum (o-1.8) and a maximum. The sexual function total score was consist of the sum of the scores of all the points and was ranged from $2-36$. The cut-off point score for the sexual function was 26.5 (Wiegel et al., 2005). The Reliability and validity of the protocol were already tested by Mohammadi et al. (2005) in Iran.

The state-trait Anxity Invertory: anxiety was measured by means of Spielberger state-trait Anxiety Inventory, which includes 40 items. The questionnaire has two parts: the first part, the result of individual anxiety status (Manifest anxiety) which includes 20 questions with a rating scale (even, to some extent, medium, very much), the second part, the result of Hidden anxiety status that includes 20 questions with a rating scale (almost no, sometimes, often, almost ever). The 4-point scale items use a score ranging from o (absence) to 3 (severe). The cutoff scores for anxiety were as follows o-20, mild; 21-40, moderate and $>60$, severe $(25,26,27)$.

Statistical analysis: collected data were analyzed by using the SPSS software v. 21. To describe the data, we used descriptive statistics, including measures of central tendency and dispersion and frequency distribution. Inferential statistics (the chi-square test, paired t-test, and one-way ANOVA) were used to analyze the collected data.

\section{Results:}

No statistical differences were revealed for demographic data (Table-1). Nevertheless, the results in Table-2 show the mean scores of the domains of sexual function (desire, excitement, orgasm, lubrication and dyspareunia), increased significantly in both the intervention groups three months after training. But in the control group, no changes occurred in the mean scores.

Table- 3 reveal that the mean overt and covert anxiety decreased significantly in both the intervention groups three months after training, while in the control group, no changes occurred in the mean scores. Moreover, the results showed that in this study, no statistically significant difference was observed between the two mentioned training methods (personal training and booklet training) on anxiety and sexual function of the subjects. (Table-4).

Table-1: Demographics of the infertile women in the control and experimental groups. Mean $\pm \mathrm{SD}$ or $\mathrm{n}(\%)(\mathrm{n}=37$ each $)$

\begin{tabular}{|c|c|c|c|c|}
\hline Training (groups)- & Personal & Control & Via Booklet & 'p' \\
\hline \multicolumn{5}{|l|}{ Age Factors } \\
\hline Woman's age & $26.43 \pm 3.89$ & $27.91 \pm 4.43$ & $26.94 \pm 5 \cdot 5^{2}$ & $\geq 0.38$ \\
\hline Husband's age & $30.89 \pm 4.16$ & $31.91 \pm 3.36$ & $31.94 \pm 6.43$ & $\geq 0.56$ \\
\hline $\begin{array}{l}\text { Age difference } \\
\text { in the couples }\end{array}$ & $5.83 \pm 4.72$ & $5 \cdot 70 \pm 4.25$ & $5.08 \pm 3.13$ & $\geq 0.69$ \\
\hline Educational level & (1) & $12(22,4)$ & $8(2,6)$ & Primary \\
\hline Secondary school & $17(45 \cdot 9)$ & $16(43.2)$ & $19(51.4)$ & $\geq 0.88$ \\
\hline Academic & $9(24 \cdot 3)$ & $9(24 \cdot 3)$ & $10(27)$ & \\
\hline \multicolumn{5}{|c|}{ Employment status } \\
\hline Housewife & $30(81.1)$ & $30(81.1)$ & $33(89.2)$ & $\geq 0.55$ \\
\hline Employed & $7(18.9)$ & $7(18.9)$ & $4(10.8)$ & \\
\hline \multicolumn{5}{|c|}{ Husband's Education } \\
\hline Primary school & $14(37.8)$ & $20(54.1)$ & $17(45 \cdot 9)$ & $\geq 0.32$ \\
\hline Secondary school & $15(40.5)$ & $15(40.5)$ & $15(40.5)$ & \\
\hline Academic & $8(21.6)$ & $2(5 \cdot 4)$ & $5(13.5)$ & \\
\hline \multicolumn{5}{|c|}{ Husband's Occupation } \\
\hline Employed & $30(81.1)$ & $30(81.1)$ & $34(91.9)$ & \\
\hline Unemployed & $7(18.9)$ & $7(18.9)$ & $3(8.1)$ & $\geq 0.32$ \\
\hline
\end{tabular}

Table-3: Comparison of the mean differences between the tree groups' anxiety scores. Mean \pm SD

\begin{tabular}{llll}
\hline Group & Pre-test & Post-test & p-value* \\
\hline State Anxiety & & & \\
Control & $50.16 \pm 3.96$ & $49.32 \pm 4.11$ & $\geq 0.10$ \\
Personal training & $50.10 \pm 3.98$ & $47.78 \pm 3.33$ & $<0.001$ \\
Via booklet training & $50.32 \pm 5.58$ & $48.89 \pm 5.53$ & $<0.0001$ \\
Trait Anxiety & & & \\
Control & $50.00 \pm 4.45$ & $48.45 \pm 3.82$ & $<0.05$ \\
Personal training & $50.32 \pm 5.01$ & $47.97 \pm 4.10$ & $<0.004$ \\
Via booklet training & $50.91 \pm 5.15$ & $49.05 \pm 4.76$ & $<0.0001$ \\
\hline
\end{tabular}

*paired t-test between the two groups' anxiety scores

Table-4: Comparison of the mean differences after training between the two groups' anxiety and female sexual function index scores. Mean \pm SD

\begin{tabular}{llll}
\hline Variable & Personal & Via booklet & p value* \\
\hline State Anxiety & $47.78 \pm 3.33$ & $48.89 \pm 5.53$ & $\geq 0.30$ \\
Trait Anxiety & $47.97 \pm 4.10$ & $49.05 \pm 4.76$ & $\geq 0.29$ \\
Total sexual function & $19.70 \pm 2.17$ & $\mathbf{2 0 . 1 7 \pm 2 . 3 7}$ & $\geq 0.37$ \\
\hline
\end{tabular}

* $\mathrm{p}$-value: Student t-test between the two intervention groups.

\section{Discussion:}

We compared the effect of infertility treatment using personal (face-to-face) training and training via booklet distribution methods on anxiety levels and sexual function among infertile women and is among the first studies that have been done in the field of educating such group of women in Iran. Based on the results of this study and comparison of averages before the intervention and 3 months after the intervention on two groups of infertile 
Table 2: Comparison between the means of the groups' scores in the various domains of sexual function before and after the intervention, Mean \pm SD ( $n=37$ in each group)

\begin{tabular}{lllllllll}
\hline Group & Control & & \multicolumn{3}{c}{ Personal training } & \multicolumn{3}{c}{ Via booklet training } \\
Sexual function domains & Pre-test & Post-test & p value* & Pre-test & Post-test* & p value & Pre-test & Post-test \\
p value*
\end{tabular}

${ }^{*}$ P-value: paired t-test between groups FSFI score, before and after the intervention

women, a significant reduction in anxiety and an increase in sexual function scores between the two groups were evident that were statistically significant. While no significant difference was observed in the control group before and after the intervention.

No similar study that compared the two methods of education and examined the impact of training on sexual function and anxiety in infertile women was yet seen. Some studies have investigated only the effectiveness of education and sexual hygiene on the sexual activity and satisfaction of pregnant women and others have explored the influence of intervention and consultation with different approaches on sexual satisfaction, intercourse and relationship quality of normal couples and nonpregnantwomen.

In order to compare the two methods of training, the study of Khadmyan et al. (2013) comparing the training methodology in terms of patients' learning levels after the urine surgeries showed that no statistically significant difference was observed between the face-to-face (personal) training and via booklet training with respect to the rate of learning in both mentioned training methods. While Comparing two methods of face-to-face or group education on sexual function of pregnant women, Bahadorani et al. (2015) showed that education had a positive impact on sexual function in both groups, but, there were no differences in sexual performance between the two teaching methods was evidenced. Riyazi et al. (2013) demonstrated that sex education with an educational booklet, plus a one-hour face-to-face training session in 6o pregnant women had a significant positive effect on the correction of wrong and dysfunctional beliefs about sex during pregnancy and on improving the quality of sex in pregnant women. These studies were consistent with our study. Our results also showed that education has a significant effect on increasing sexual performance and on reducing anxiety, and secondly, via booklet training also revealed a positive impact similar to the personal method. The findings suggest that the patients' lack of knowledge and awareness about infertility treatments can have a direct relationship with anxiety and sexual disorders so that after training, patients have experienced less anxiety and sexual dysfunction.

Following the intervention of various methods with stress and anxiety in infertile women, the results of our study are strengthening the following studies. Studying the effectiveness of group counseling, based on a participatory approach, on proprietary stress in infertile women, Rabeipour et al. (2016) reported a significant difference in overall stress scores after the intervention between the intervention and control groups. In the study of Latifnejad et al. (2011) infertility overall stress was reduced at the end of the study and the results of Sexton et al. (2010) showed that online coping with the infertility stress leads to reducing general stress symptoms in infertile women. Valiani et al. (2015) referred to the role of relaxation techniques to reduce stress and anxiety in infertile women and showed that anxiety scores in the intervention and control groups were significantly different and the intervention resulted in anxiety reduction. The results of all studies pointed towards the role of intervention in reducing anxiety in infertile women that are consistent with our study in this respect. Further, our study also showed that intervention through the two aforementioned methods and providing appropriate training and the necessary information about the physiology of the reproductive system, causes of Infertility, treatment process could greatly reduce the anxiety of women during infertility treatment.

In addition, our results showed that both interventional methods in the present study have a significant and positive impact on the areas of sexual function (libido, orgasm, dyspareunia, sexual satisfaction). There was no statistically signif icant difference between the two methods of intervention.

Studies by Vural \& Temel (2009) \& Rostamkhani et al. (2012) showed a significant increase in the female libido in the intervention group that was consistent with our study. The results of the study conducted by Ayaz \& Kubilay (2009) showed that consultation on the training program has significant effects of on women's orgasm. The results Choobforoushzadeh et al. (2010) on the effectiveness of stress management therapy in a cognitive-behavioral manner in connubial satisfaction in infertile women also indicated that connubial satisfaction mean scores in the 
experimental group in post-test and follow-up increased significantly compared to those of control group. Miri et al. (2016) examined the impact of an enrichment relations educational program of the compatibility and connubial satisfaction in infertile couples and found that average score of compatibility and connubial satisfaction and couple's agreement had increased in the intervention group. Our study strengthening the above findings in terms of the effect of intervention on enhancing connubial performance and satisfaction. Psychological disorders such as stress in the vicious cycle that they created compounded the problems. Using such training, infertile people can strengthen their family by taking advantage of desirable connubial satisfaction and a life without stress and worry. Obviously, the presence of resources and gynecologists' teamwork, counseling in obstetrics and psychology in infertility clinics would perform a positive role in reducing the problems of such people.

\section{Conclusion:}

According to the findings of this study, it can be concluded that applying both approaches of face-to-face training and booklet training increases sexual performance and reduces anxiety. However, in comparing the two intervention groups, no significant difference was found. So, despite the effectiveness of in-person training and advantages such as the presence of the trainer and interaction with patients, the findings of this study showed that despite the lack of an educating and active element, booklet training has been effective in increasing sexual function in patients and in reduc ingtheir anxiety. So acknowledging the causes of infertility and available treatments can be effective in increasing sexual performance and reducing anxiety in infertile people. This training can lead to better understanding of people about sexual issue sand to learning the proper way to deal with stressful situations during this period and, ultimately to improving mental health. Therefore, counseling programs and sex education seem essential as a part of infertility treatment.

Inspite of the large size of the sample, one of the strengths of our study some shortcomings existed. The absence of the participants' husbands in the educational sessions was one of them. Raising the awareness of infertile women's husbands could also play a role in increasing the satisfaction of the former. Some of the patients were unaware of their psychological problems and the need for them to consult a psychologist or psychiatrist and were therefore unwilling to participate in the study. In Iran, sex education is often a hard job to do due to the current religious and cultural taboos, thus it is important that people's cultural values and needs be considered in the development of such programs. The women whose husbands had a sexual dysfunction or who had a sexually transmitted disease were not excluded from the study, which could be had influenced the findings of the study.
Raised awareness of women in the study, who used sources other than the intended curriculumwhich in turn may have affected the results of the present study,was amongthe limitations of the present study. Crises and potential problems for the individuals and their family emerged in the research,individual differences and the patient's mental states were among the limitations of this study.

\section{Acknowledgment:}

Thanks are due towards Jahrom University of Medical Sciences for approving the protocol of this study and financial support.

\section{References:}

Akhondi, M.M., Kamali, K., Ranjbar, F., Shirzad, M., Shafeghati, S., Behjati Ardakani, Z., Goodjani, A., Parsaeian, M. \& Mohammad, K. (2013): Prevalence of Primary Infertility in Iran in 2010. Iran J Public Health, 42(12):1398-1404.

Ayaz, S. \& Kubilay, G. (2008): Effectiveness of the PLISSIT model for solving the sexual problems of patients with stoma. J. Clin. Nurs., 18(1):89-98.

Bahadorani, P., Mahdiabadzade, M., Nasiri, H. \& GholamiDehaghi, A. (2015): The effect of face-to-face or group education during pregnancy on sexual function of couples in Isfahan. Iran. J. Nurs. Midwifery Res., 20(5):582-587.

Baldur-Felskov, B., Kjaer, S.K., Albieri, V., Steding-Jessen, M., Kjaer, T., Johansen, C., Dalton, S.O. \& Jensen, A. (2013): Psychiatric disorders in women with fertility problems: results from a large Danish register-based cohort study. Hum Reprod., 28(3):683-69o.

Bastable, S.B. (2013): Nurse as Educator: Principles of Teaching and Learning for Nursing Practice. (4th Ed) Pub. by: Jones \& Bartlett Learning. 716 p.

Chehreh, H. Neisani Samani, L., Seyedfatemi, N. \& Hosseini, A.F. (2012): Anxiety and its Relationship with Infertility and Obstetrics Factors in ART Pregnant Women. Iran. J. Nursing, 25(77):77-84.

Choobforoushzadeh, A., Kalantari, M., Molavi, H. (2010): The effectiveness of cognitive behavioural stress management therapy on marital satisfaction in infertile women. J. Fundam. Ment. Health. 12(3):596-603.

Ebrahimipour, H., Jalambadany, Z., Peyman, N., Ismaili, H. \& Najjar, A.V. (2013): Effect of sex education, based on the theory of planned behavior, on the sexual function of the woman attending Mashhad health centers. J. Birjand Uni. Med. Sci., 20(1):58-67.

Hamid, N. (2011): The Effectiveness of Stress Management Based on Depression, Anxiety and Fertilization of Infertile Women. $]$. Behav. Sci., 5 (1):15-16. (Persian).

Jamali, S., Zarei, H. \& Jahromi, A.R. (2014): The relationship between body mass index and sexual function in infertile women: A cross-sectional survey. Iran. J. Reprod. Med., 12(3).189-198.

Kashdan, T.B., Adams, L., Savostyanova, A., Ferssizidis, P., Macknight, P.E. \& Nezlek, J.B. (2011): Effects of social anxiety and depressive symptoms on the frequency and quality of sexual activity: A daily process approach. Behav Res Ther., 49(5):352-36o. 
Khadmyan, Z., Magharei, M., Shokraniyan, N. (2013): Comparison of two methods of face to face training and instruction booklet on learning after surgery, urinary tract. Nur. Edu., 1(1):70-77.

Kring, A.M. \& Johnson, S.L. (2017): Abnormal Psychology. 14th ed. Pub. by: Wiley, New York.

Latifnejad R.R, Rasolzadeh, B.M, Mousavifar, N. \& Modarres, G.M. (2011): The effect of collaorative counseling on perceived infertility-related stress in infertile women undergoing IVF. Women Obstetric. Infert. Iran, 14(4):22-31.

Mirblouk, F., Asgharnia, D.M., Solimani, R., Fakor, F., Salamat, F. \& Mansoori, S. (2013): Comparison of sexual dysfunction in women with infertility and without infertility referred to AlZahra Hospital in 2013-2014. Int. J. Reprod. Biomed. (Yazd). 14(2):117-124.

Miri, M., Alizadeh, M., Moasheri, N., Ataei, M. \& Modi, M. (2016): The effects of relationship enrichment program on compatibility and marital satisfaction of infertile couples. $L$ Health Lit.,1(1):53-60.

Mousavi S, Sabzevari S, Abbaszade A, Hosseinnakhaie F. (2011): The effect of preparatory face to face education to reduce depression and anxiety in open heart surgery adult patient in Shafa hospital in Kerman. Iran. J. Nursing Res., 6(21):29-38.

Onat, G. \& Beji, N.K. (2012): Marital relationship and quality of life among couples with infertility. Sex. Disabil., 30(1):39-52.

Pakpour, A.H., Yekaninejad, M.S., Zeidi, I.M., Burri, A. (2012): Prevalence and risk factor of female sexual dysfunction in a sample of infertile Iranian. Arch. Gynecol .Obstet., 286(6):1589-1596.

Perry, T.E.\& Hirshfeld-Cytron, J. (2013): Role of complementary and alternative medicine to achieve fertility in uninsured patients. Obstet. Gynecol. Surv.,68(4):305-311.

Ramezanzadeh, F., Aghssa, M.M., Abedinia, N., Zayeri, F., Khanafshar, N., Shariat, M. \& Jafarabadi, M. (2004): A survey of relationship between anxiety, depression and duration of infertility. BMC Womens Health, 4(1):9.
Rabeipour, S., Avval, Z.O., Arefi, M. \& Behroozilak, T. (2016): Effectiveness of group counseling based on a participatory approach, proprietary stress in infertile women: a randomized clinical trial. J. Urmia Nurs. Midwif.,14(1):56-65.

Riazi H, Banoo, Z.S., Moghim, B.A. \& Amini, L. (2013): The effect of sexual health education on sexual function during pregnancy. Payesh, 12(4):367-374. (Persian).

Rostamkhani, F., Ozgoli, G., Khoei, E.M., Jafari, F. \& Majd H.A. (2012): Effectiveness of the PLISSIT-based Counseling on sexual function of women. Adv. Nurs. Midwif., 22(76):1-9.

Shakarami, M., Davarniya, R., Zahrakar, K. \& Gohari, Sh. (2014): The effect of sex education on sexual intimacy of married women.Iran.J. Psych. Nur., 2(1):34-42.

Sexton MB, Byrd MR, O’Donohue WT, Jacobs NN. (2010): Webbased treatment for infertility-related psychological distress. Arch. Womens Ment. Health, 13(4):347-58.

Tavakolizadeh, J. \& Hajivosogh N.S. (2012): The Effect of Cognitive Behavioral Teaching on Marital Satisfaction of Women With Hypoactive Sexual Desire Disorder. Sci. J. Ilam Uni. Med. Sci., 21(5):44-50.

Tie, S. \& Poulsen, S.S. (2013): Emotionally focused couple therapy with couples marital adjustment. Contemp. Fam. Ther., 35(3):557-567.

Valiani M, Abedian S. Ahmadi, S. \& Pahlavanzade, S. (2015): Infertile women's satisfaction with the stress reduction care process during their treatment period: A clinical trial. Sci.J. Hamedan Nurs. Midwif. Fac., 23(1):38-48. (Persian).

Vural, B.K. \& Temel, A.B. (2009): Effectiveness of premarital counseling program on sexual satisfaction of recently married couples. Sex Health. 6(3):222-232.

Yousefzadeh, S., Nameni, F., Golmakani, N., Najafi, M.N., Ebrahimi, M., Gharavi, M.M. (2014): Investigating the Effects of Sex Education Based on Religious Education on Marital Adjustment in Married Women. Iran. J. Obst. Gynecol. Infert.,16(84):10-19 